\title{
Effect of Fenofibrate on Microcirculation and Wound Healing in Healthy and Diabetic Mice
}

\author{
S. Valentin ${ }^{1}$, J. Rudolph ${ }^{1}$, O. Goertz ${ }^{2}$, N. Botteck ${ }^{2}$, S. Langer ${ }^{2}$, S. Schneider ${ }^{1}$ \\ ${ }^{1}$ Medical Department I, University Hospital Bergmannsheil, University of Bochum, Germany; \\ ${ }^{2}$ Department of Plastic and Hand Surgery, University Hospital Bergmannsheil, University of Bochum, Germany
}

\begin{abstract}
Objective: Disturbances in wound healing in patients with hyperglycaemic blood sugar values are a common clinical problem. Recent studies identified PPAR $\alpha$-ligands as potential skin therapeutic agents. The aim of this study was to investigate the effects of oral fenofibrate treatment on dermal wound healing and microcirculatory parameters in diabetic mice.

Methods: Dermal wounds were created in CD-1 mice. Mice were randomized into four treatment groups: diabetic mice treated (dbf) or not-treated with fenofibrate (dbnf). As controls served non-diabetic mice treated (ndf) or not-treated with fenofibrate (ndnf). At various points in time microcirculation was analyzed by intravital fluorescent microscopy to determine wound surface area, vessel diameter, plasma leakage, functional capillary density, and leukocyte/endothelium interaction.

Results: The dbf-mice showed a significantly increased diameter of the venules and the arterioles up to 3 days after wound creation compared to dbnf-mice. However, wound healing was not improved in dbf-compared to dbnf-mice. Surprisingly, all microcirculatory parameter (vessel diameter, plasma leakage and functional capillary density) were not deteriorated in dbnf- compared to ndnf-mice.

Conclusion: We confirm that high blood sugar values lead to a delayed wound healing, but this could not traced back to altered microcirculatory patterns. Furthermore, in dbf-mice an improved vasodilatatory function of small vessels could be detected, but with no substantial effect on wound healing. Further studies are needed to clarify, if topical application of fenofibrate might be beneficial.
\end{abstract}

Key words: wound healing, fenofibrate, microcirculation, diabetes, peroxisome proliferator activated receptor (PPAR)

\section{INTRODUCTION}

Diabetic ulcers complications are a leading cause of hospitalization and amputation. Ten to $15 \%$ of the 20 million individuals with diabetes are at risk of developing diabetic ulcers (Centers of Disease Control and Prevention 1998). Standard therapy involves the use of dressings to protect the wound bed from trauma and to absorb exsudate, offloading of high pressure on the wound bed e.g. by prescribing protective footwear, and wound bed preparation to accelerate endogenous healing. However these measures are often deficient to heal all diabetic ulcers when the patient's own intrinsic wound healing system is insufficient (Falanga et al. 2005). Therefore, the search for new targets and their synthetic ligands which improve wound healing are highly recommended.

In recent years several studies support a link between the 3 peroxisome proliferator activated receptors (PPAR $\alpha, \delta$, and $\gamma$ ) and diabetes, obesity, dyslipidemia, and inflammation. Increased recognition of a role for PPARs in metabolic- and inflammation regulation came following the discovery of fibrates and thiazolidinediones were synthetic ligands for PPAR $\alpha$ and PPAR $\gamma$ (Issemann et al. 1990, Formann et al. 1997, Lehmann et al. 1995). PPAR $\alpha$ influences intracellular lipid and carbohydrate metabolism through direct transcriptional control of genes involved in peroxisomal and mitochondrial $\beta$-oxidation pathways, uptake of fatty acids, and triglyceride catabolism. Furthermore, PPAR $\alpha$ agonists inhibit the expression of several inducible factors which are implicated in endothelial, macrophage and smooth muscle cell function (Lefebvre et al. 2006). Moreover, PPAR $\alpha$ activation results in an anti-inflammatory action as demonstrated in the PPAR $\alpha$-deficient mouse model (Devchand et al. 1996). Clinically, these molecular actions of PPAR $\alpha$ activators culminate in a reduction of circulating inflammatory markers and a reduction of the progression of atherosclerotic lesions (Lefebvre et al. 2006). The ability of PPAR $\alpha$ to improve metabolic alterations suggests that PPAR $\alpha$ may be beneficial in the prevention or treatment of patients with diabetes mellitus and their associated complications. Therefore, we tested the effect of the PPAR $\alpha$ agonist fenofibrate on wound healing and circulatory parameters in healthy and diabetic mice.

\section{Materials AND Methods}

Animals: Male CD1 mice (26-38 g body weight) were obtained from Charles River, Sulzfeld, Germany. The animals had access to standard laboratory feed (18000 
IE/kg Vit. A, 1280 IE/kg Vit. D3, 120 mg/kg Vit. E; sniff, Spezialdiaeten, Soest, Germany) and tap water ad libitum. The mice were housed individually at $21{ }^{\circ} \mathrm{C}$ temperature in a 12 hour dark/12 hour light cycle. The principles of laboratory animal care were followed (Ruhr-University Bochum, No.105/6). For the experiments, mice were anesthetized by spontaneous inhalation of isoflurane-N2O (FiO2 0.35, 0.015 L/L Isoflurane, Forene, Abbott $\mathrm{GmbH}$, Wiesbaden, Germany) and placed on a heated custom made observation platform to maintain body temperature at $37.0^{\circ} \mathrm{C}$.

Induction of diabetes: Under general anaesthesia mice were assigned up to three intraperitoneal injections on day $-14,-11$ and -7 of $60 \mathrm{mg} / \mathrm{kg}$ streptozotocin $(\mathrm{N}$ Methylnitrosocarbamoyl-D-glucosamine; Sigma, Deisenhofen, Germany) per kg body weight to induce diabetes. The induction of diabetes was carried out 2 weeks prior to the start of the experiments. Blood glucose level was determined by means of analysis of tail vein blood every day after the first application (akkucheck). Animals with blood glucose levels over $350 \mathrm{mg} / \mathrm{dl}$ were accepted for the study.

Hair depilation on mouse ear: In order to get good and analysable microcirculatory records we depilated mouse hair on the ears with pilca depilation crème on day $-1,+6,+13$ and +20 . The wound area was protected with tegaderm foil during depilation so that it did not get in contact with the crème.

Wound creation: Wounds were created as described previously (Langer et al. 2002, Langer et al. 2004). Briefly, the area selected for wounding on the dorsal side of the ear was marked with an O-shaped ink stamp of a diameter of $2.5 \mathrm{~mm}$. Using this mark, a full-thickness dermal layer down to the underlying cartilage was excised by means of microsurgical scissors (Medicon, Tuttlingen, Germany) under a surgical microscope. This preparation provided a full-thickness dermal wound of approx. $5 \mathrm{~mm}^{2}$ with a depth of approx. $120 \mu \mathrm{m}$.

Application of fenofibrate: Primarily, we examined the minimal and maximal eating dose of a representative group of ten diabetic and non-diabetic mice. The minimal eating dose was $3 \mathrm{~g}$. In order to get sure that a mice with a minimal weight of $25 \mathrm{~g}$ gets its total daily dose, fenofibrate $(10 \mathrm{mg} / \mathrm{kgKG} / \mathrm{d})$ was mixed into normal laboratory mice food $(18000 \mathrm{IE} / \mathrm{kg}$ Vit. A, $1280 \mathrm{IE} / \mathrm{kg}$ Vit. D3, $120 \mathrm{mg} / \mathrm{kg}$ Vit. E; sniff, Spezialdiaeten, Soest, Germany) in a concentration of 0.0833 mg per gram food.

Experimental groups: Mice were randomized into four treatment groups: diabetic mice treated (dbf, $\mathrm{N}=10$ ) or not-treated with fenofibrate (dbnf, $\mathrm{N}=9$ ). As controls served non-diabetic mice treated (ndf, $\mathrm{N}=12$ ) or not-treated with fenofibrate (ndnf, $\mathrm{N}=10$ ).

Measurement using intravital fluorescent microscopy: Following exteriorization of the ear by means of three permanent suture loops the animal was placed on a computer controlled stage to perform the investiga- tions with an intravital fluorescent microscope (Harris et al. 1997). A 4-fold and a 20-fold water immersion objective (Zeiss Axiotech, Zeiss, Oberkochen, Germany) was used, resulting in total magnifications of 57 -fold (wound surface measurements). The X-Y coordinates of the region of interests (ROI) were recorded (AxioVision 3.0, Zeiss, Germany) and their position stored on the computer to ensure relocation of identical areas throughout the study. In addition, drawings of the microcirculatory areas were made to ensure relocation of the ROI. For contrast enhancement of the plasma $0.1 \mathrm{ml}$ of $2 \%$ fluorescein isothiocyanate (FITC) labeled Dextran (MW 150,000; Sigma Chemicals Co., St. Louis, USA) was injected prior to the measurements. For visualization of leukocytes, the fluorescent marker Rhodamine 6G (Sigma, Chemicals Co., St. Louis, USA) was given i.v. as a bolus of 0.15 $\mathrm{mg} / \mathrm{kg} 10$ minutes before the measurements. The images were recorded using a charge-coupled device video camera (AVT-BC 71, AVT- Horn, Aalen, Germany) on super VHS video tapes (Sony, Cologne, Germany). At the end of the observations the animals were released to their cages. Microscopic observations were performed prior to wounding (day 0) and repeated on day 3, 7, 14 and 21 after wound creation. At the end of the experiments the animals were killed under general anaesthesia by an overdose of i. p. given pentobarbital.

Measurement of wound surface area: While restrained in the observation platform images of the wound surface area under the intravital microscopy setup were performed with a total magnification of 57 -fold. At this magnification the wound occupied a single microscopic field and allowed for its observation on the monitor. From these digital images the wound surface area was calculated by a digital planimetric mode integrated into the computer assisted image analysis system CapImage (Dr. Zeintl, Heidelberg, Germany) and expressed as $\mathrm{mm}^{2}$ (Klyscz et al. 1997).

Microcirculatory parameters: Microcirculatory measurements were made at 3 ROIs located at the edge of the wound. Inside those ROIs a total of 3 venules, 3 arterioles as well as 3 capillary fields per animal were recorded. The recording time for each field of observation was 20 seconds. The following parameters were analyzed: 1 . Diameter of arterioles and venules $[\mu \mathrm{m}]$, 2. Functional capillary density (FCD) $\left[\mathrm{cm} / \mathrm{cm}^{2}\right], 3$. Macromolecular leakage of the plasma marker FITCDextran from venules [Ie/Ii], 4. The number of adherent leukocytes in venules $[\mathrm{n} / 100 \mu \mathrm{m}]$. FCD is defined as the length of red blood cell perfused capillaries per observational area. This parameter is used as an index for tissue perfusion (Nolte et al. 1995). Macromolecular leakage of the plasma marker FITC-Dextran out of vessels is a measure of endothelial integrity (Harris et al. 1996). It is given as the ratio of the fluorescence intensities measured outside vs. inside a vessel. According to their interaction with the endothelium, leukocytes were defined as adherent in the case they did not move from microvascular endothelium during the observation time of 20 seconds (Nolte et al. 1994, Terajima et al. 2000). 
Statistics: The commercially available computer program SigmaStat (Jandel Scientific, Erkrath, Germany) was used for statistical analysis of the data. Data is given as mean \pm standard deviation (sd). Comparison between groups was performed using a non-parametric Kruskal-Wallis test followed by Dunn's method. A $\mathrm{p}<0.05$ was considered statistically significant.

\section{RESUlTS}

Blood glucose values and body weight: Neither the nondiabetic- (ndnf vs. ndf) nor the diabetic (dbnf vs. ndf) groups showed a difference concerning their blood glucose values. These results suggest that fenofibrate hasn `t an impact on blood glucose control (Fig. 1).



Fig. 1. Blood glucose values were significantly higher in dbf(white triangle) and dbnf-mice (black triangle) compared to ndf- (black circle) and ndnf-mice (white circle) over the whole study period, but a substantial effect of fenofibrate treatment on blood glucose control could not be observed. Values are represented mean \pm sd.

Wound surface area: The diabetic-mice showed irrespective of their treatment alignment a significantly worsened wound healing compared to their nondiabetic controls (Fig. 2). At day 21 only two-thirds of the wound area were closed in the diabetic mice whereas in the non-diabetic mice the wound was almost completely closed. These results suggest that wound healing problems exist at early stages of diabetes development, keeping in mind that diabetes was induced by streptozotocin only two weeks prior the examination. Furthermore, the wound surface area was compared among the nondiabetic mice (ndf-mice vs. ndnf-mice), with a tendency for a faster wound healing in the ndf-mice, but a significant difference could not be detected at any time point. The same holds true for the dbfmice when compared to the dbnf-mice. These observations suggest that fenofibrate treatment leads in our model not to an improvement of macroscopic wound healing neither in non-diabetic nor in diabetic mice.

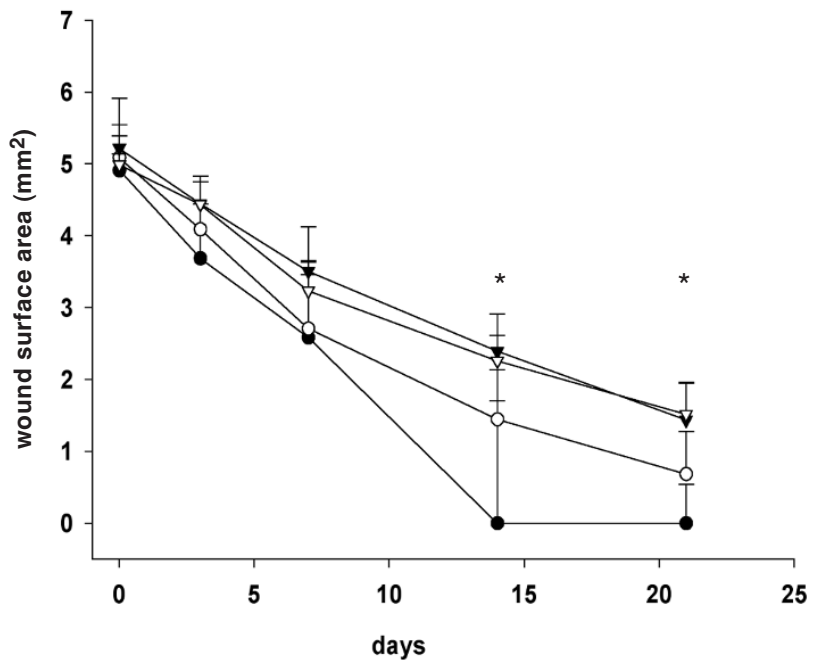

Fig. 2. Wound surface area was significantly higher in dbf(white triangle) and dbnf-mice (black triangle) compared to ndf- (black circle) and ndnf-mice (white circle) at day 14 and 21 , but a significant effect of fenofibrate treatment on macroscopic wound healing could not be observed. Values are represented mean + sd. $* \mathrm{p}<0.05$

Microcirculatory parameters (Table 1): The arteriolar diameter increased significantly from day 0 to 3 in the dbf-mice, and decreased thereafter to the starting value. In contrast, the arteriolar diameter of the other groups (ndnf, ndf, dbnf) remained nearly unchanged. Furthermore, the dbf-mice showed a significant vasodilatation of the venules between day 0 and day 3 , whereas in the other groups such an effect could not be observed. In addition, in all groups the FCD increased significantly until day 7 , but with no difference among the groups. Furthermore, a significant accumulation of leukocytes could be observed in all experimental groups. The rolling leukocytes occurred in a larger number on day 3 and the sticky leukocytes could be detected in a larger amount on day 3 and 7. Concerning the endothelial leakage a significant venous extravasation could be observed in all groups on day 3 . In both diabetic groups there was a tendency to more diffusion of the macromolecular plasma marker FITC-Dextran, but this remained not statistical significant (data not shown).

\section{Discussion}

This study demonstrates an improvement of endothelial dysfunction in diabetic mice treated with fenofibrate (dbf-mice). However, an effect on wound healing and blood glucose control could not be demonstrated.

It has been shown that hyperglycaemic blood glucose levels lead to an altered lipid profile including an elevation of triglycerides and free fatty acids (FFA), which in turn are toxic to endothelial cells leading to vascular dysfunction (Steinmetz 2003, Mittermayer et al. 2007). Fibrates are a widely used class of lipid-regulating agents, exerting a variety of effects on lipid- and lipoprotein metabolism, particularly reduction of triglycerides and FFA (Staels et al. 1998). In our study 
Table 1. Microcirculatory parameters of the 4 different treatment groups at predefined points in time.

\begin{tabular}{|c|c|c|c|c|c|}
\hline Parameter & day & ndf & ndnf & $\mathrm{dbf}$ & dbnf \\
\hline \multirow{5}{*}{$\mathrm{FCD}\left(\right.$ in $\left.\mathrm{cm} / \mathrm{cm}^{2}\right)$} & 0 & $187.2 \pm 18.2$ & $199.0 \pm 11.4$ & $190.8 \pm 14.1$ & $199.5 \pm 8.7$ \\
\hline & 3 & $215.5 \pm 16.6$ & $210.8 \pm 15.8$ & $201.9 \pm 12.7$ & $211.0 \pm 10.3$ \\
\hline & 7 & $229.5 \pm 14.5^{\mathrm{x}}$ & $222.1 \pm 13.9^{\mathrm{X}}$ & $212.0 \pm 10.9^{\mathrm{X}}$ & $215.8 \pm 6.8^{x}$ \\
\hline & 14 & $214.5 \pm 19.5$ & $209.4 \pm 8.9$ & $205.4 \pm 10.6$ & $208.1 \pm 9.4$ \\
\hline & 21 & $195.1 \pm 14.6$ & $202.4 \pm 11.6$ & $195.1 \pm 12.0$ & $198.3 \pm 9.6$ \\
\hline \multirow[t]{5}{*}{ Diameter of arterioles (mm) } & 0 & $41.0 \pm 12.3$ & $43.5 \pm 8.3$ & $37.7 \pm 2.9$ & $42.0 \pm 6.6$ \\
\hline & 3 & $41.0 \pm 9.2$ & $45.6 \pm 10.5$ & $45.5 \pm 3.8 \times$ & $44.2 \pm 7.8$ \\
\hline & 7 & $40.9 \pm 8.1$ & $46.6 \pm 10.3$ & $41.6 \pm 2.5$ & $45.5 \pm 5.0$ \\
\hline & 14 & $39.6 \pm 10.4$ & $45.1 \pm 10.0$ & $40.2 \pm 2.9$ & $42.7 \pm 5.4$ \\
\hline & 21 & $42.8 \pm 12.8$ & $43.4 \pm 9.6$ & $40.1 \pm 2.4$ & $42.0 \pm 6.3$ \\
\hline \multirow[t]{5}{*}{ Diameter of venules (mm) } & 0 & $82.8 \pm 18.3$ & $82.8 \pm 12.1$ & $83.9 \pm 7.8$ & $78.5 \pm 10.3$ \\
\hline & 3 & $85.5 \pm 22.3$ & $85.8 \pm 15.4$ & $94.7 \pm 8.1^{*}$ & $81.1 \pm 13.9$ \\
\hline & 7 & $82.4 \pm 19.6$ & $83.6 \pm 16.3$ & $90.3 \pm 8.4$ & $76.7 \pm 13.0$ \\
\hline & 14 & $83.3 \pm 17.1$ & $83.9 \pm 13.3$ & $86.1 \pm 8.2$ & $75.6 \pm 11.2$ \\
\hline & 21 & $80.8 \pm 20.2$ & $81.7 \pm 12.2$ & $85.0 \pm 7.8$ & $77.1 \pm 9.7$ \\
\hline \multirow{5}{*}{$\begin{array}{l}\text { Rolling leukocytes } \\
\text { (n/100mm/20sec) }\end{array}$} & 0 & $11.2 \pm 2.1$ & $12.0 \pm 3.1$ & $12.5 \pm 2.0$ & $13.0 \pm 1.5$ \\
\hline & 3 & $14.2 \pm 1.9^{\mathrm{X}}$ & $15.5 \pm 2.5^{x}$ & $15.4 \pm 1.4 \mathrm{x}$ & $16.0 \pm 1.1^{\mathrm{x}}$ \\
\hline & 7 & $11.8 \pm 1.7$ & $13.1 \pm 1.9$ & $12.7 \pm 1.2$ & $13.2 \pm 1.5$ \\
\hline & 14 & $11.5 \pm 1.8$ & $11.6 \pm 2.7$ & $11.9 \pm 0.9$ & $12.1 \pm 1.3$ \\
\hline & 21 & $11.0 \pm 1.5$ & $11.6 \pm 2.4$ & $12.1 \pm 0.9$ & $12.0 \pm 0.9$ \\
\hline Sticky leukocytes & 0 & $3.1 \pm 0.8$ & $3.6 \pm 1.2$ & $4.0 \pm 1.2$ & $3.8 \pm 0.7$ \\
\hline \multirow[t]{4}{*}{$(\mathrm{n} / 100 \mathrm{~mm} / 20 \mathrm{sec})$} & 3 & $4.9 \pm 1.4 \mathrm{X}$ & $5.9 \pm 0.9^{x}$ & $5.4 \pm 1.1^{\mathrm{X}}$ & $5.3 \pm 1.0^{\mathrm{X}}$ \\
\hline & 7 & $4.8 \pm 0.8^{x}$ & $6.1 \pm 1.2^{\mathrm{x}}$ & $4.6 \pm 0.8^{x}$ & $5.2 \pm 1.1 \mathrm{X}$ \\
\hline & 14 & $3.9 \pm 0.7$ & $5.1 \pm 1.9$ & $3.9 \pm 0.7$ & $4.6 \pm 0.5$ \\
\hline & 21 & $3.5 \pm 1.0$ & $4.0 \pm 1.7$ & $3.6 \pm 1.0$ & $3.8 \pm 0.8$ \\
\hline
\end{tabular}

$\mathrm{x}<0.05$ vs. baseline, $* \mathrm{p}=0.007$ vs. baseline. Mean $\pm \mathrm{sd}$.

we detected in the dbf-mice a significant increased diameter of the venules as well as of the arterioles up to 3 days after wound creation compared to dbnf-mice. This effect seems not be a direct interaction of fenofibrate with PPAR $\alpha$-receptors in the vessel wall, because in ndf-mice such an effect could not be observed. The mechanisms by which fenofibrate leads to an improved vasodilatation remains to be speculative, but there are good clinical data that fibrates improve endothelial dysfunction in patients with type 2 diabetes by modifying hyperglycaemia-induced atherogenic lipid profile (reduction of FFA and triglycerides, increased HDL-C) and reduction of oxidative stress (Evans et al. 2000).

Wound repair requires the integration of interdependent processes and signals that involve, among others, soluble mediators, inflammatory cytokines produced by a variety of cell types, cell proliferation and migration, cell differentiation and production of extracellular matrix components. The hypothesis that PPAR $\alpha$ participates in the control of the inflammatory process during wound repair is reinforced by the observation that PPAR $\alpha-/-$ mice show an impaired wound healing due to uncontrolled inflammation at wound site (Michalik et al. 2001). Furthermore, it has been shown that topical application of a PPAR $\alpha$-agonist to mice with hyperproliferative skin disorders normalizes cell proliferation and promotes epidermal differentiation and correct the cutaneous pathology (Kömüves et al. 2000). Therefore, PPAR $\alpha$-activators have been identified as potential skin therapeutic agents. However, in our study systemic application of fenofibrate by oral gavage in a therapeutical dose improved neither in the diabetic nor in the non-diabetic mice the wound healing process significantly. Therefore, further studies are necessary to clarify if topical application of fenofibrate or other PPAR $\alpha$-activators may improve wound healing in diabetic animals.

The wound healing process was significantly impaired in the diabetic mice compared to the non-diabetic control mice, despite diabetes was induced only 2 weeks prior starting the experiments. These results are in agreement with those obtained by others (Langer et al. 2002). Surprisingly, the impaired wound healing could not traced back to an altered microcirculation, because all the examined parameter (vessel diameter, red blood cell velocity, plasma leakage and functional capillary density) were not deteriorated in the dbnfmice compared to the ndnf-mice. These results suggest that the impaired wound healing process in mice 
with short diabetes duration is primarily a problem of the local wound environment. Recently, it has been shown that some of the resident cells in diabetic ulcers are phenotypically altered. Fibroblasts isolated from diabetic foot ulcers are probably senescent and show a decreased proliferative response to growth factors (Loot et al. 2002). Similar studies in other types of wounds are in agreement with these findings, having shown decreased fibroblast response to TGF $\beta 1$, platelet-derived growth factor, and other cytokines (Hasan et al. 1997, Agren et al. 1999, Stanley et al. 2005). Evidence suggests that phenotypic changes in wound cells are not due only to replicative senescence, but are perhaps caused by more complex interactions between the resident cells and the wound (Stephens et al. 2003). Wound fibroblasts do show decreased expression of type 2 TGF $\beta$ receptors, with impaired phosphorylation of transduction signals, including Smad2, Smad3, and mitogen-activated protein kinase (Kim et al. 2003). Although much more work is needed to clearly define the phenotypical abnormalities in diabetic wound cells, these findings have clear-cut implications for therapeutic intervention. For example, growth factors delivered in a simplistic topical approach might not find a regularly suitable and responsive target cell population.

Other cellular abnormalities exist in diabetes. Excessive activation of some MMPs, such as MMP9, can impair cell migration and lead to breakdown of some necessary matrix proteins and growth factors (Signorelli et al. 2005). Although there is no direct evidence that the proliferative activity of keratinocytes is affected in diabetes, migration may well be impaired and studies with cells from diabetic wounds are needed (Acikgoz et al. 2004). Therefore, beside correction of hyperglycemia, proper debridement of diabetic ulcers corrects many more subtle abnormalities, at least partly, by removal of altered resident cells and matrix material.

In summary, we confirm that that high blood sugar values lead in a very short time frame to an impaired wound healing process, but this alteration could not traced back to altered microcirculatory patterns. Furthermore, in diabetic mice systemic fenofibrate treatment leads to an improved vasodilatatory function of small vessels in the early phase of wound healing (first 3 days). However, this corrects not the delayed wound healing in diabetic mice. Further studies are needed to clarify, if topical application of fenofibrate might improve wound healing.

Acknowledgements: This work was supported by the Bergmannsheil Förderung (Forum 05-Innere-533).

\section{REFERENCES}

1. Acikgoz G, Devrim I, Ozdamar S. Comparison of keratinocyte proliferation in diabetic and non-diabetic inflamed gingiva. Aggressive periodontitis with supernumerary teeth: a retrospective study. J Periodontol. 2004 Nov; 75(11):1458-60.

2. Agren MS, Steenfos HH, Dabelsteen S, Hansen JB, Dabelsteen E. Proliferation and mitogenic response to PDGF-BB of fbroblasts isolated from chronic venous leg ulcers is ulcer-age dependent. J Invest Dermatol. 1999 Apr; 112(4):463-9.
3. Bondar I, Uhl E, Barker JH, Galla TJ, Hammersen Fand Messmer K. A new model for studying microcirculatory changes during dermal wound healing. Res Exp Med (Berl). 1991; 191(6):379-88

4. Centers for Disease Control and Prevention. Diabetes-related amputations of lower extremities in the Medicare population - Minnesota, 1993-1995. MMWR Morb. Mortal. Wkly. Rep. 1998; 47:649-652.

5. Devchand PR, Keller H, Peters JM, Vazquez M, Gonzalez FJ, Wahli W. The PPAR $\alpha$-leukotriene B-4 pathway to inflammation control. Nature. 1996 Nov 7;384(6604): 3943.

6. Falanga V. Wound healing and its impairement in the diabetic foot. Lancet. 2005 Nov 12; 366(9498): 1736-43.

7. Forman BM, Chen J, Evans RM. Hypolipidemic drugs, polyunsaturated fatty acids and eicosanoids are ligands for peroxisome proliferator-activated receptors a and $\mathrm{d}$. Proc. Proc Natl Acad Sci U S A. 1997 Apr 29; 94(9): 4312-7.

8. Harris AG, Hecht R, Peer F, Nolte D, Messmer K. An improved intravital microscopy system. Int J Microcirc Clin Exp. 1997 Nov-Dec; 17(6): 322-7.

9. Harris AG, Leiderer R, Peer F, Messmer K. Skeletal muscle microvascular and tissue injury after varying durations of ischemia. Am J Physiol. 1996 Dec; 271(6 Pt 2): 238898.

10. Hasan A, Murata H, Falabella A, Ochoa S, Zhou L, Badiavas E, Falanga V. Dermal fibroblasts from venous ulcers are unresponsive to the action of transforming growth factor-beta1. J Dermatol Sci. 1997 Nov; 16(1): 59-66.

11. Issemann I, Green S. Activation of a member of the steroid hormone receptor superfamily by peroxisome proliferators. Nature. 1990 Oct 18; 347(6294): 645-50.

12. Kim BC, Kim HT, Park SH, Cha JS, Yufit T, Kim SJ, Falanga V. Fibroblasts from chronic wounds show altered TGF-beta-signaling and decreased TGF-beta Type II receptor expression. J Cell Physiol. 2003 Jun; 195(3): 331-6.

13. Klyscz T, Jünger M, Jung $F$ and Zeintl $H$. Capimage-a new kind of computer-assisted video image analysis system for dynamic capillary microscopy. Biomed Tech (Berl). 1997 Nov; 42(11): 310-8.

14. Kömüves LG, Hanley K, Man MQ, Elias PM, Williams ML, Feingold KR. Keratinocyte differentiation in hyperproliferative epidermis: topical application of PPARalpha activators restores tissue homeostasis. J Invest Dermatol. 2000 Sep; 115(3): 361-64

15. Langer S, Born F, Breidenbach A, Schneider A, Uhl E, Messmer K. Effect of C-peptide on wound healing and microcirculation in diabetic mice. Eur J Med Res. 2002 Nov 25; 7(11): 502-8.

16. Langer S, Sedigh Salakdeh M, Goertz O, Steinau HU, Steinstraesser L, Homann HH. The impact of topical antiseptics on skin microcirculation. Eur J Med Res. 2004 Sep 29; 9(9): 449-54. 17. Lefebvre P, Chinetti G, Fruchart J-C and Staels B. Sorting out the roles of PPAR $\alpha$ in energy metabolism and vascular homeostasis. J Clin Invest. 2006 Mar; 116(3): 571-80.

18. Lehmann JM, Moore LB, Smith-Oliver TA, Wilkison WO, Willson TM, Kliewer SA. An antidiabetic thiazolidinedione is a high affinity ligand for peroxisome proliferator-activated receptor g. J Biol Chem. 1995 Jun 2; 270(22): 12953-6.

19. Loot MA, Kenter SB, Au FL, van Galen WJ, Middelkoop E, Bos JD, Mekkes JR. Fibroblasts derived from chronic diabetic ulcers differ in their response to stimulation with EGF, IGF-I, bFGF and PDGF-AB compared to controls. Eur J Cell Biol. 2002 Mar; 81(3): 153-60

20. Terajima H, Enders G, Thiaener A, Hammer C, Kondo T, Thiery J, Yamamoto Y, Yamaoka Y, Messmer K. Impact of hyperthermic preconditioning on postischemic hepatic microcirculatory disturbances in an isolated per- 
fusion model of the rat liver. Hepatology. 2000 Feb; 31(2): 407-15.

21. Michalik L, Desvergne B, Tan NS, Basu-Modak S, Escher P, Rieusset J, Peters JM, Kaya G, Gonzalez FJ, Zakany J, Metzger D, Chambon P, Duboule D, Wahli W. Impaired skin wound healing in peroxisome proliferator-activated receptor (PPAR)alpha and PPARbeta mutant mice. J Cell Biol. 2001 Aug 20; 154(4): 799-814.

22. Mittermayer F, Schaller G, Pleiner J, Krzyzanowska K, Kapiotis S, Roden M, Wolzt M. Rosiglitazone prevents free fatty acid-induced vascular endothelial dysfunction. J Clin Endocrinol Metab. 2007 Jul; 92(7): 2574-80.

23. Nolte D, Schmid P, Jager U, Botzlar A, Roesken F, Hecht $\mathrm{R}$, Uhl E, Messmer K, Vestweber D. Leukocyte rolling in venules of striated muscle and skin is mediated by P-selectin, not by L-selectin. Am J Physiol. 1994 Oct; 267: 1637-42.

24. Nolte D, Zeintl H, Steinbauer M, Pickelmann S, Messmer $\mathrm{K}$. Functional capillary density: an indicator of tissue perfusion? Int J Microcirc Clin Exp. 1995 Sep-Oct; 15(5): 244-9.

25. Signorelli SS, Malaponte G, Libra M, Di Pino L, Celotta G, Bevelacqua V, Petrina M, Nicotra GS, Indelicato M, Navolanic PM, Pennisi G, Mazzarino MC. Plasma levels and zymographic activities of matrix metalloproteinases 2 and 9 in type II diabetics with peripheral arterial disease. Vasc Med. 2005 Feb; 10(1): 1-6.

26. Stanley AC, Fernandez NN, Lounsbury KM, Corrow K, Osler T, Healey C, Forgione P, Shackford SR, Ricci MA. Pressure-induced cellular senescence: a mechanism linking venous hypertension to venous ulcers. J Surg Res. 2005 Mar; 124(1): 112-7.

27. Steinmetz A. Treatment of diabetic dyslipoproteinemia. Exp Clin Endocrinol Diabetes. 2003 Aug; 111(5): 239-45.
28. Stephens P, Cook H, Hilton J, Jones CJ, Haughton MF, Wyllie FS, Skinner JW, Harding KG, Kipling D, Thomas DW. An analysis of replicative senescence in dermal fbroblasts derived from chronic leg wounds predicts that telomerase therapy would fail to reverse their diseasespecifc cellular and proteolytic phenotype. Exp Cell Res. 2003 Feb 1;283(1):22-35.

29. Terajima H, Enders G, Thiaener A, Hammer C, Kondo T, Thiery J, Yamamoto Y, Yamaoka Y, Messmer K. Impact of hyperthermic preconditioning on postischemic hepatic microcirculatory disturbances in an isolated perfusion model of the rat liver. Hepatology. 2000 Feb; 31(2): 407-15.

30. Uhl E, Sirsjo A, Haapaniemi T, Nilsson G, Nylander G. Hyperbaric oxygen improves wound healing in normal and ischemic skin tissue. Plast Reconstr Surg. 1994 Apr; 93(4): 835-41.

Received: February 7, 2008 / Accepted: June 19, 2008

Address for correspondencer:

Stephan Schneider, MD

Medical Department 1

Division of Endocrinology and Metabolism

University Hospital Bergmannsheil

Ruhr-University of Bochum

Bürkle de la Camp Platz 1

44789 Bochum

Germany

Phone: +49-234-302-3469

Fax:+49-234-302-6415

E-mail: Stephan.Schneider@ruhr-uni-bochum.de 